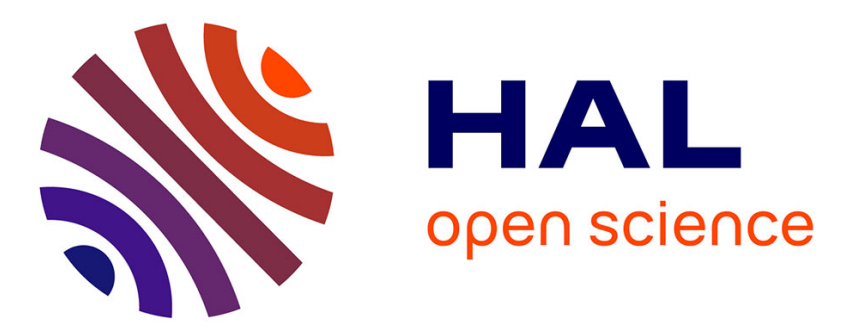

\title{
Mean and median-based nonparametric estimation of returns in mean-downside risk portfolio frontier
}

Hanene Ben Salah, Mohamed Chaouch, Ali Gannoun, Christian de Peretti, Abdelwahed Trabelsi

\section{- To cite this version:}

Hanene Ben Salah, Mohamed Chaouch, Ali Gannoun, Christian de Peretti, Abdelwahed Trabelsi. Mean and median-based nonparametric estimation of returns in mean-downside risk portfolio frontier. Annals of Operations Research, 2018, 262 (2), pp.653-681. 10.1007/s10479-016-2235-z . hal01300673

\section{HAL Id: hal-01300673 https://hal.science/hal-01300673}

Submitted on 12 Apr 2016

HAL is a multi-disciplinary open access archive for the deposit and dissemination of scientific research documents, whether they are published or not. The documents may come from teaching and research institutions in France or abroad, or from public or private research centers.
L'archive ouverte pluridisciplinaire HAL, est destinée au dépôt et à la diffusion de documents scientifiques de niveau recherche, publiés ou non, émanant des établissements d'enseignement et de recherche français ou étrangers, des laboratoires publics ou privés. 


\title{
Mean and median-based nonparametric estimation of returns in mean-downside risk portfolio frontier
}

\author{
Hanene Ben Salah • Mohamed Chaouch • Ali \\ Gannoun · Christian de Peretti · Abdelwahed \\ Trabelsi
}

Received : date / Accepted : date

Résumé The DownSide Risk (DSR) model for portfolio optimisation allows to overcome the drawbacks of the classical Mean-Variance model concerning the asymmetry of returns and the risk perception of investors. This model optimization deals with a positive definite matrix that is endogenous with respect to portfolio weights. This aspect makes the problem far more difficult to handle. For this purpose, Athayde (2001) developed a new recursive minimization procedure that ensures the convergence to the solution. However, when a finite number of observations is available, the portfolio frontier presents some discontinuity and is not very smooth. In order to overcome that, Athayde (2003) proposed a Mean Kernel estimation of the returns, so as to create a smoother portfolio frontier. This technique provides an effect similar to the case in which continuous observations are available. In this paper, Athayde model is reformulated and clarified. Then, taking advantage on the robustness of the median, another nonparametric approach based on Median Kernel returns estimation is proposed in order to construct a portfolio frontier. A new version of Athayde's algorithm will be exhibited. Finally, the properties of this improved portfolio frontier are studied and analysed on the French Stock Market.

Keywords DownSide Risk · Kernel Method · Mean Nonparametric Estimation · Median Nonparametric Estimation · Portefolio Efficient Frontier · Semi-Variance.

\section{H. Ben Salah}

BESTMOD Laboratory, ISG 41 Rue de la Liberté, Le Bardo,Tunisie.

Institut de Science Financière et d'Assurances, LSAF EA2429, F-69366 Lyon, France.

IMAG 34095 Montpellier cedex 05, France

E-mail: bensalah_hanen@yahoo.fr

M. Chaouch

Department of Statistics, United Arab Emirates University, UAE.

E-mail: m.chaouch@uaeu.ac.ae

\section{A. Gannoun}

IMAG, 34095 Montpellier cedex 05, France

E-mail: ali.gannoun@um2.fr

C. de Peretti

Univ Lyon, Université Claude Bernard Lyon 1,

Institut de Science Financière et d'Assurances, LSAF EA2429, F-69366 Lyon, France.

E-mail: Christian.De-Peretti@ec-lyon.fr

A. Trabelsi

BESTMOD Laboratory, ISG 41 Rue de la Liberté, Le Bardo,Tunisie.

E-mail: abdel.trabelsi@gmail.com 


\section{Introduction}

Optimizing asset allocation is simply defined as the process of mixing asset weights of a portfolio within the constraints of an investor's capital resources to yield the most favourable risk-return trade-off. For typical risk-averse investors, an optimal combination of investment assets that gives a lower risk and a higher return is always preferred (see Markowitz (1952)). In a complete market without riskless lending and borrowing, a whole range of efficient asset portfolios having the stochastic dominance features could be determined, which collectively delineates a convex Mean-Variance frontier.

The classical Mean-Variance (M-V) portfolio optimization model introduced by Markowitz (1952) aims at determining the proportions $\omega_{i}$ of a given capital to be invested in each asset $i$ belonging to a predetermined set or market so as to minimize the risk of the return of the whole portfolio for specified expected return. The risk is measured by the variance. More precisely, suppose that $m$ assets are available, and denote by $\mu_{i}$ the expected return of asset i , and $\sigma_{i j}$ the Covariance of returns of assets $i$ and $j$ for $i, j=1, \ldots, m$. Let $E^{*}$ be the required level of return for the portfolio, the classical M-V model is the following optimization program :

$$
\begin{gathered}
\min _{\omega_{1}, \omega_{2}, \ldots, \omega_{m}} \sum_{i=1}^{m} \sum_{j=1}^{m} \omega_{i} \omega_{j} \sigma_{i j} \\
\text { subject to } \sum_{i=1}^{m} \omega_{i} \mu_{i}=E^{*} \quad \text { and } \quad \sum_{i=1}^{m} \omega_{i}=1 .
\end{gathered}
$$

This is a convex quadratic programming problem which can be solved by a number of efficient algorithms with a moderate computational effort even for large instance. This problem can be solved for a specific value of $E^{*}$ or, alternatively, for several values of $E^{*}$ thus generating the minimum variance set. Either way, it is important to notice, firstly, that the risk of the portfolio can be expressed as a function of the risk of the individual assets in the portfolio; secondly, that all the Variances, Covariances, and Expected Returns of the individual assets are exogenous variables.

However, the use of variance as a risk measure is a questionable measure of risk for at least three reasons : (1) it makes no distinction between gains and losses, (2) it is an appropriate measure of risk only when the underlying distribution of returns is symmetric and (3) it can be applied as a risk measure only when the underlying distribution of returns is Normal.

Markowitz (1959) recognised the "asymmetrical" inefficiencies inherited in the traditional M-V model. To overcome the drawbacks of this model, he suggested to use a DownSide Risk (DSR) measured by

$$
\mathrm{DSR}=\frac{1}{T} \sum_{t=1}^{\top}\left[\min \left(r_{p t}-B, 0\right)\right]^{2},
$$

where $r_{p t}$ denotes the returns of the portfolio and $B$ any benchmark return chosen by the investor. The benchmark can be equal to 0 (or the risk-free rate), any stock market index (CAC 40) or the mean $\mu$ of the portfolio return. Note that if $B=\mu$, the DSR is the so called Semivariance. The DSR is a more robust measure of asset risk that focuses only on the risks below a target rate of return. This measure of risk is a more plausible for several reasons. Firstly, investors obviously do not dislike upside volatility; they only dislike downside volatility, secondly, the DSR is more useful than the variance when the underlying distribution of returns is asymmetric and just as useful when the underlying distribution is symmetric; in other words, the DSR is at least a measure of risk as useful as the variance, and finally the DSR combines into one measure the information provided by two statistics, variance and skewness, thus making it possible to use a 
one-factor model to estimate required returns. Then, the optimization model can be written as follows :

$$
\begin{gathered}
\min _{\omega_{1}, \omega_{2}, \ldots, \omega_{m}} \frac{1}{T} \sum_{t=1}^{T}\left[\min \left(r_{p t}-B, 0\right)\right]^{2} \\
\text { subject to } \sum_{i=1}^{m} \omega_{i} \mu_{i}=E^{*} \quad \sum_{i=1}^{m} \omega_{i}=1 .
\end{gathered}
$$

However, finding the portfolio with minimum DSR is not an easy task. The major obstacle to get the solution of this problem is that the Semicovariance matrix is endogenous; that is, a change in weights affects the periods in witch the portfolio under-performs the target rate of return, which in turn affects the element of the Semicovariance matrix.

Many approaches are suggested to estimate the portfolio semivariance. For example, Markowitz (1959) suggested the following estimator :

$$
\Sigma_{p B}^{2}=\sum_{i=1}^{m} \sum_{j=1}^{m} \omega_{i} \omega_{j} \Sigma_{i j B}, \quad \text { where } \quad \Sigma_{i j B}=\frac{1}{T} \sum_{t=1}^{K}\left(r_{i t}-B\right)\left(r_{j t}-B\right),
$$

where periods 1 to $K$ are periods in witch the portfolio under-performs the benchmark $B$. The advantage of this estimator is that provides an exact estimation of the portfolio Semivariance. However, the Semicovariance matrix remains endogenous. Many authors propose different ways to resolve problem (3). Among them, Hogan and Warren (1974) proposed to use the the Frank-Wolf algorithm. Ang (1975) proposed to linearise the Semivariance so that the optimization problem can be solved using linear programming. Harlow (1991) also considered problem (3) and generated Mean-Semivariance efficient frontier, which he compares to the Mean-Variance efficient frontiers. Markowitz et al. (1993) transformed the Mean-Semivariance problem into a quadratic problem by adding fictitious securities. King (1993) extended the Levy-Markowitz argument (Levy and Markowitz, 1979) to account for asymmetric risk by basing the local approximation on piecewise linear-quadratic risk measures, which can be tuned to express a wide range of preferences and adjusted to reject outliers in the data. Mamoghli and Daboussi (2008) improved Harlow approach and their model allows to overcome the problem of inequality of the Semicovariance measures which occur in the Mean-Semivariance model of Harlow. Estrada (2008) proposed a simple and accurate heuristic approach that yields a symmetric and exogenous Semicovariance matrix, which enables the determination of Mean-Semivariance optimal portfolios by using the well known closed-form solutions of Mean-Variance problem. Athayde (2003) generalised his own iterative algorithm developed in (2001) to construct a Mean-DownSide risk portfolio frontier. The major contribution in Athayde (2001) is to replace returns by their mean kernel estimations (nonparametric mean regressions). The great advantage of this technique is to provide an effect similar to the case in which observations are continuous. The new portfolio frontier has a smoother shape than the traditional one. Although Athayde contribution is innovative, his two papers are unstructured with no simulations and no applications. Another neglected aspect which deserves serious attention concerns the theory : a great confusion is palpable in the estimators writing.

In this paper, Athayde's work is first revisited by making it rigorous. The mean nonparametric estimator is clarified and its parameters are exhibited as well as their practical choices. Than the corresponding optimization algorithm is coded using R-software and empirically validated on real data. Secondly, taking advantage on the robustness of the median, Athayde's work is improved by proposing another method to optimize a portfolio : this new method is based on nonparametric 
estimation of conditional median based on kernel method. It is well-known that the median is more robust than the mean and less sensitive to the outliers. Returns will be replaced by their nonparametric median estimators. Additionally, the optimal portfolio will be obtained under positivity constraints (all the $\omega_{i}$ are positive) and Short Selling constraints $\left(\omega_{i} \in \mathbb{R}\right)$. In order to validate this method, a new algorithm is written and coded. All the optimization methods are compared to each others and their efficiency is checked against the CAC40 index and using Sharp and Sortino ratios.

The paper is organised as follows. Section 2 presents the classical concepts of the DSR and the Athayde's algorithm optimisation. A general presentation of the nonparametric estimators is given in Section 3. In Section 4, the previous algorithm is extended to incorporate nonparametric returns estimation, based on conditional mean and conditional median approaches, in the DSR measures. Section 5 is devoted to a real data analysis of the French Stock Market. Finally, some conclusions and perspectives are discussed in Section 6 .

\section{DSR : the traditional approach}

The aim of this section is to present the algorithm of Athayde. The purpose of this algorithm is to give an iterative solution to Downside optimization problem which deals with a positive definite matrix. This matrix is endogenous to portfolio weights. This aspect makes the problem far more difficult to handle. For this purpose, Athayde (2001) developed a simple algorithm that ensures the convergence to the solution. Below, we outline this algorithm. We deal with a 2-asset portfolio analysis in 2.1 and present general $m$-asset case in 2.2 .

2.1 Case of a portfolio with two assets

\subsubsection{Formal framework}

To fix ideas, assume two risky assets $a$ and $b$ are available. The return of a portfolio $p$ that has $\omega$ units of $a$ and $(1-\omega)$ units of $b$, at time $t$ can be expressed as :

$$
r_{p t}(\omega)=\omega r_{a t}+(1-\omega) r_{b t},
$$

where $r_{i t}$ stands for the return of asset $i$ at time $t$.

Consider that observations start on date time 1 and end on date $T$,

$$
\operatorname{DSR}(\omega)=\frac{1}{T} \sum_{t=1}^{T}\left[\min \left(r_{p t}(\omega)-B, 0\right)\right]^{2} .
$$

The value of $\omega$ at time $t$ that makes the portfolio's return equal to the benchmark $B$ is given by :

$$
\omega_{t}=\frac{B-r_{b t}}{r_{a t}-r_{b t}} .
$$

If only this observation is available, assuming that $r_{a t}>r_{b t}$, the DSR of this portfolio, respectively its first and second derivative with respect to $\omega$, would be given by : 


$$
\left\{\begin{aligned}
\operatorname{DSR}(\omega) & =\left[\omega\left(r_{a t}-r_{b t}\right)-\left(B-r_{b t}\right)\right]^{2} \text { if } \omega<\omega_{t} \\
& =0 \text { otherwise } \\
\operatorname{DSR}^{\prime}(\omega) & =2\left[\omega\left(r_{a t}-r_{b t}\right)-\left(B-r_{b t}\right)\right] \times\left(r_{a t}-r_{b t}\right)<0 \text { if } \omega<\omega_{t} \\
& =0 \text { otherwise } \\
\operatorname{DSR}^{\prime \prime}(\omega) & =2\left(r_{a t}-r_{b t}\right)^{2}>0 \text { if } \omega<\omega_{t} \\
& =0 \text { otherwise }
\end{aligned}\right.
$$

The symmetric case, i.e when $r_{a t}<r_{b t}$, is treated similarly.

Remark 1 To compute the DSR as a function of the weight $\omega$, the piecewise quadratic functions (when $r_{a t}>r_{b t}$ and when $r_{a t}<r_{b t}$ ) should be added . Every time, crossing points like $\omega_{t}$ implies a change in the convexity of the curve. Including all observations, the whole DSR will be a curve as shown in Figure 1. In the figure 1 (a), the DSR is a function of weight $\omega$. In the figure 1 (b), the DSR is a function of expected return $E\left(r_{p}\right)$. The two curves are similar because that the expected portfolio return is a linear function of $\omega$ (see (9)).

FiguRe 1: DSR curves.

The expected return of the portfolio will be given by :

$$
E\left(r_{p}\right)=\omega E\left(r_{a}\right)+(1-\omega) E\left(r_{b}\right) \Leftrightarrow \frac{E\left(r_{p}\right)-E\left(r_{b}\right)}{E\left(r_{a}\right)-E\left(r_{b}\right)}=\omega
$$

From the linear relation between $\omega$ and $r_{p}$ :

$$
E\left(r_{p}\right)=\omega\left(E\left(r_{a}\right)-E\left(r_{b}\right)\right)+E\left(r_{b}\right),
$$

the shape of the set DSR $\times E\left(r_{p}\right)$ is drawn in Figure 1 .

As it has been shown, this curve is a sum of segments of quadratic functions. The curve will become steeper and steeper as we move toward the extremes, in either directions. The more observations we have, the more quadratic functions will be added and smaller the segment of each will become. The changes in the convexity, when we move from one quadratic function to another will become more frequent and smoother. In the limit case, where we will have an infinite number of observations, each of these quadratic functions will degenerate to a single point, creating a continuous smooth changing in the convexity of the curve. Thus, in the bivariate case, the portfolio set and consequently the portfolio frontier will have a convex shape.

\subsubsection{The algorithm}

The goal here is to find the portfolio weights that give the minimum DSR, that is the vertex of the curve above should be determined. To this end, Athayde's algorithm, which is summarized below, is used : 
step 1 : Start with an arbitrary portfolio $\omega_{0}$ and compute its DSR. Then construct the set of date $S_{0}$ such that

$$
S_{0}=\left\{t \mid 1 \leq t \leq T \text { and } r_{p t}\left(\omega_{0}\right)<B\right\}
$$

and consider the following curve

$$
\sigma_{0}^{2}(\omega)=\frac{1}{T} \sum_{t \in S_{0}}\left(r_{p t}(\omega)-B\right)^{2}, \text { where } r_{p t}(\omega)=\omega r_{a t}+(1-\omega) r_{b t} \text {. }
$$

Note that, to start the process, $\omega_{0}=(1 / 2,1 / 2)$ is used.

step 2 : Minimize $\sigma_{0}^{2}(\omega)$ over $S_{0}$ to get $\omega_{1}=\arg \min _{\omega} \sigma_{0}^{2}(\omega)$. This is an ordinary quadratic problem getting a minimum at

$$
\omega_{1}=\frac{\sum_{t \in S_{0}}\left(r_{a t}-r_{b t}\right)\left(B-r_{b t}\right)}{\sum_{t \in S_{0}}\left(r_{a t}-r_{b t}\right)^{2}}
$$

calculate its DSR. Construct the set $S_{1}$ such that

$$
S_{1}=\left\{t \mid 1 \leq t \leq T \text { and } r_{p t}\left(\omega_{1}<B\right\}\right.
$$

and consider the following quadratic curve

$$
\sigma_{1}^{2}(\omega)=\frac{1}{T} \sum_{t \in S_{1}}\left(r_{p t}(\omega)-B\right)^{2}, \text { where } r_{p t}(\omega)=\omega r_{a t}+(1-\omega) r_{b t}
$$

step 3 : Minimize $\sigma_{1}^{2}(\omega)$ over $S_{1}$ to get $\omega_{2}=\arg \min _{\omega} \sigma_{1}^{2}(\omega)$ which is :

$$
\omega_{2}=\frac{\sum_{t \in S_{1}}\left(r_{a t}-r_{b t}\right)\left(B-r_{b t}\right)}{\sum_{t \in S_{1}}\left(r_{a t}-r_{b t}\right)^{2}},
$$

calculate its DSR. Construct the set $S_{2}$ such that

$$
S_{2}=\left\{t \mid 1 \leq t \leq T \text { and } r_{p t}\left(\omega_{2}\right)<B\right\}
$$

step 4 : Using $S_{2}$, like the previous steps, determine $\omega_{3}$ that will give a new set $S_{3}$, and so on. The algorithm will stop after $F$ iterations. It happens when $S_{F}=S_{F+1}$ and then the optimal portfolio weights are

$$
\omega_{F, a}=\frac{\sum_{t \in S_{F}}\left(r_{a t}-r_{b t}\right)\left(B-r_{b t}\right)}{\sum_{t \in S_{F}}\left(r_{a t}-r_{b t}\right)^{2}}, \quad \omega_{F, b}=1-\omega_{F, a} .
$$

2.2 Case of a portfolio with $m$ assets

In this section, the bivariate case is extrapolated to the multivariate one. The focus is only on the minimization algorithm. First, the Multivariate DownSide Risk portfolio is presented, then the portfolio frontier is exhibited. 


\subsubsection{The algorithm}

Say that $m$ assets are available, let $R_{j t}=r_{j t}-B$ the excess return of the asset $j$ on the date $t$ where $B$ the chosen benchmark.

A given portfolio $\omega_{0}$ is used for starting. A set $S_{0}$ of observations is selected in which the portfolio $\omega_{0}$ had negative deviations. The following positive semi-definite matrix is then constructed :

$$
\begin{array}{r}
M_{0}=\frac{1}{T} \sum_{t \in S_{0}}\left[\begin{array}{c}
R_{1 t} \\
R_{2 t} \\
\vdots \\
R_{m t}
\end{array}\right]\left[\begin{array}{lll}
R_{1 t} & R_{2 t} \cdots R_{m t}
\end{array}\right] \\
=\frac{1}{T} \sum_{t \in S_{0}}\left[\begin{array}{ccc}
\left(R_{1 t}\right)^{2} & R_{1 t} R_{2 t} & R_{1 t} R_{m t} \\
R_{2 t} R_{1 t} & \left(R_{2 t}\right)^{2} & R_{2 t} R_{m t} \\
\vdots & \vdots & \vdots \\
R_{m t} R_{1 t} & R_{m t} R_{2 t} & \left(R_{m t}\right)^{2}
\end{array}\right] .
\end{array}
$$

The next step is to find the portfolio $\omega_{1}$ that solves the following problem :

$$
\min _{\omega} \omega^{\top} M_{0} \omega \text { with } \omega^{\top} \mathbf{1}=1
$$

where $\mathbf{1}$ is a vector of 1 s and $\omega^{\top}$ the transpose of the vector $\omega$.

Using Lagrangian Method (see Annexe 1), the solution to the problem will be given by ${ }^{1}$

$$
\omega_{1}=\frac{M_{0}^{-1} \mathbf{1}}{\mathbf{1}^{\top} M_{0}^{-1} \mathbf{1}} .
$$

With the new portfolio $\omega_{1}$, a set $S_{1}$ of observations that contains only negative excess returns of portfolio $\omega_{1}$ is determined. A new positive semidefinite matrix $M_{1}$ is then constructed :

$$
M_{1}=\sum_{t \in S_{1}}\left[\begin{array}{ccc}
\left(R_{1 t}\right)^{2} & R_{1 t} R_{2 t} & R_{1 t} R_{m t} \\
R_{2 t} R_{1 t} & \left(R_{2 t}\right)^{2} & R_{2 t} R_{m t} \\
\vdots & \vdots & \vdots \\
R_{m t} R_{1 t} & R_{m t} R_{2 t} & \left(R_{m t}\right)^{2}
\end{array}\right] .
$$

The next step is to find the portfolio $\omega_{2}$ that solves the following problem :

$$
\min _{\omega} \omega^{\top} M_{1} \omega \text { such that } \omega^{\top} \mathbf{1}=1 \text {. }
$$

As previously, the solution to the problem will be given by :

$$
\omega_{2}=\frac{M_{1}^{-1} \mathbf{1}}{\mathbf{1}^{\top} M_{1}^{-1} \mathbf{1}} .
$$

Then, the previous process is iterated to construct a sequence of matrices $M_{i}$ until getting the first matrix $M_{F}$ satisfying the criterion $M_{F}=M_{F+1}$. The optimal portfolio will be given by :

$$
\omega_{F+1}=\frac{M_{F}^{-1} \mathbf{1}}{\mathbf{1}^{\top} M_{F}^{-1} \mathbf{1}} .
$$

1. If $M_{0}$ is non-invertible, this means that we will have few observations, and that it will be possible to find a portfolio that will give us a null DSR. This is not an interesting case, it does not mean there is no DSR, it only means that the sample in question is poor, leaving us with few degrees of freedom. 
This portfolio will give the minimum DSR.

Remark 2 It is clear that there are no constraints on the signs of weights $\omega_{i, 1}, \omega_{i, 2}, \ldots, \omega_{i, m}$ which make up the vector $\omega_{i}$ (on the step $i-1$ ). These constraints will be introduced in next sections. This is one of the contributions that make this work different from Athayde's one (2003).

\subsubsection{The portfolio frontier}

In terms of the portfolio frontier, the DSR will represent the vertex of the curve. In order to build the portfolio frontier, some other points on the efficient set should be found. Since the interest is in points with a higher expected return than the vertex, an expected return $E^{*}$ a bit higher than the minimum DownSide risk portfolio above should be fixed. So the new recursive minimization procedure will take the following form :

$$
\min _{\omega} \omega^{\top} M \omega \text { such that } \omega^{\top} \mathbf{1}=1 \text { and } \omega^{\top} \mu=E^{*},
$$

where $\mu$ is the vector of expected returns and $E^{*}$ the target return of the portfolio.

$F$ iterations, with the Lagrangian Method Optimisation at each iteration, are used to achieve the convergence. The minimum DownSide Risk Portfolio weights with expected excess return $E^{*}$ will be given by :

$$
\omega_{F+1}=\frac{\alpha E^{*}-\lambda}{\alpha \theta-\lambda^{2}} M_{F}^{-1} \mu+\frac{\theta-\lambda E^{*}}{\alpha \theta-\lambda^{2}} M_{F}^{-1} \mathbf{1},
$$

where $\alpha=\mathbf{1}^{\top} M_{F}^{-1} \mathbf{1}, \lambda=\mu^{\top} M_{F}^{-1} \mathbf{1}$ et $\theta=\mu^{\top} M_{F}^{-1} \mathbf{1}$.

The minimum DSR is obtained by pre-multiplying $\omega_{F}^{\top} M_{F}(25)$ :

$$
\operatorname{DSR}\left(\omega_{F+1}\right)=\frac{\alpha\left(E^{*}\right)^{2}-2 \lambda E^{*}+\theta}{\alpha \theta-\lambda^{2}} .
$$

Remark 3 The equation (19) shows that while the final matrix $M_{F}$ does not change, DownSide risk will be a quadratic (parabola) function on the expected return, just like the bivariate case. However, if the expected return changes considerably, this leads to a new final matrix, and therefore a new quadratic function, because new values for $\alpha, \lambda$ and $\theta$ are got. The optimization algorithm given by (18) does not take into account the sign of the weights $\omega_{i, 1}, \omega_{i, 2}, \ldots, \omega_{i, m}$. A more efficiency analytic optimization program will be proposed.

The portfolio frontier will be described as a sequence of segments of different quadratic functions. This result is expected because the portfolio frontier is a convex combination of several bivariate cases (see above). To get a smoother frontier, more and more assets should be used, creating a similar effect as if we were adding more observations. Nonparametric technique allows to create a similar effect to those of continuous observations. Therefore, one can build a new portfolio frontier with a smoother shape, and avoid huge changes in the convexity.

\section{DSR : The kernel approach}

Using the classical mean-semivariance method, the portfolio frontier presents some discontinuity on its convexity. This is due to the fact that there are a finite number of observations. In order to overcome that, we will make use of a more sophisticated estimation of DSR, in which the 
density of the returns is estimated using kernels. A kernel estimation of one point can be seen as a weighted average or median of the observations, in which the weight given to each observation decreases with its distance from the point in question. The initial idea was given by Athayde in two papers dating back to (2001) and (2003). However, in both the papers, the estimators have been badly exposed with several mistakes and imprecisions. The parameters and indices were not clear with confused notation.

In this section, we clarify all the Athayde's notation, exhibit properly the kernel mean estimator and define and expand an alternative kernel median estimator with is more robust than Athayde's one. This section starts by an overview on nonparametric estimation where an adequate estimator and the corresponding DSR are exposed.

\subsection{Basic Background}

First meaning of nonparametric covers techniques that do not rely on data belonging to any particular distribution. In particular, they may be applied in situations where less is known about the application in question.

In the following, a return kernel density estimate is proposed. Kernel mean estimation of one point can be seen as a weighted average of the observations, in which the weight given to each observation decreases with its distance from the point in question.

First of all, some elements which will be used to define kernel estimators are introduced.

The kernel function $K(\cdot)$ : is a probability density function. For theoretical commodity, the following properties are supposed :

$$
\text { (a) } \int z K(z) d z=0 \quad \text { (b) } \int z^{2} K(z) d z<\infty .
$$

Some examples of kernels :

- Rectangular : $K(z)=\frac{1}{2}$ for $|z|<1,0$ otherwise.

- Triangular : $K(z)=1-|z|$ for $|z|<1,0$ otherwise.

- Biweight : $K(z)=\frac{15}{16}\left(1-z^{2}\right)^{2}$ for $|z|<1,0$ otherwise.

- Epanechnikov : $K(z)=\frac{3}{4}\left(1-\frac{1}{5} z^{2}\right)$ for $|z|<\frac{1}{5}$, 0 otherwise.

- Gaussian : $K(z)=\frac{1}{\sqrt{2 \pi}} \exp \left(-\frac{1}{2} z^{2}\right)$.

The bandwidth $h(T):=h$ : is a sequence of positive real numbers which decreases to 0 as $T$ tends to infinity. It is also called the the smoothing parameter.

Remark 4 It has been shown in the statistical literature that the choice of the kernel does not affect a lot the nonparametric estimation procedure. However, the choice of the bandwidth is crucial.

\subsection{Kernel mean estimation}

\subsubsection{Kernel mean return estimation}

Given $T$ return observations $r_{t}, t=1, \ldots, T$, from a given asset or portfolio, the kernel estimator $\hat{f}(\cdot)$ of probability density return $f(\cdot)$ is defined by

$$
\hat{f}\left(r_{t}\right)=\frac{1}{T h} \sum_{l=1}^{T} K\left(\frac{r_{t}-r_{l}}{h}\right) .
$$


It is characterized by the kernel $K$ which determines the shape of the weighting function, and the bandwidth $h$ which determines the width of the weighting function and hence the amount of smoothing. The two components determine the properties of the estimator. Theoretical (minimization of asymptotic errors) and practical (rule of thumb, cross validation, plug-in methods) researches had been carried out (and continue to be carried out) on the question of how one should select $K$ and $h$ in order to optimize the properties of the estimator. More details can be found in Pagan and Ullah (1999), Silverman (1986), Subramaniana (2002), Wang (2002) and Casanova and Lecompte (2015). To build a more sophisticated estimation of DSR, Athayde (2001, 2003) used the kernel estimator to replace all the observations $r_{t}, t=1, \ldots, T$ by mean kernel estimators (mean regression) $\hat{r}_{t}, t=1, \ldots, T$ which are a weighted average of the observations, in which the weight given to each observation decreases with its distance from the point in question. The estimator $\hat{r}_{t}$ of some return $r_{t}$ for a given asset or portfolio is then given by :

$$
\hat{r}_{t}=\frac{\sum_{l=1}^{T} r_{l} K\left(\frac{r_{t}-r_{l}}{h}\right)}{\sum_{l=1}^{T} K\left(\frac{r_{t}-r_{l}}{h}\right)} .
$$

\subsubsection{Kernel mean and DSR estimation}

Let $\omega=\left(\omega_{1}, \omega_{2}, \ldots \omega_{m}\right)$ be a vector of weights, using (21), a new estimation of the DSR of a given portfolio with $n$ assets, is defined as

$$
\operatorname{DSR}(\omega)=\frac{1}{T} \sum_{t=1}^{T}\left[\min \left(\hat{r}_{p t}-B, 0\right)\right]^{2}, \hat{r}_{p t}=\omega \hat{r}_{1 t}+\omega \hat{r}_{2 t}+\ldots+\omega \hat{r}_{m t},
$$

where $\hat{r}_{j t}, j=1 \ldots, m$, is the kernel mean estimator of the return $r_{j t}$ of asset $j$ on date $t$.

The construction of the algorithm to get the portfolio frontier with the new DSR, will be exhibited in Section 4. In the following, a new approach to estimate the DSR is proposed. This approach is based on kernel median estimation.

\subsection{Kernel median estimation}

\subsubsection{General concept}

While an average has traditionally been a popular measure of a mid-point in a sample, it has the disadvantage of being affected by any single value being too high or too low compared to the rest of the sample. This is why a median is sometimes taken as a better measure of a mid point. The median is generally used for skewed distributions. It is better suited for skewed distributions to derive at central tendency since it is much more robust and sensible. In regression and prediction context, the median is more robust and most adapted to model data than the mean when the conditional distribution is multi-modal or asymmetric.

From theoretical point of view, if $(X, Z)$ represents a random vector $\mathbb{R}^{2}$-valued and $F(. \mid X=x)$ the conditional distribution of $Z$ given $X=x$, the conditional median $m(x)$ of $Z$ given $X=x$ is the solution of the following minimization problem :

$$
m(x)=\arg \min _{y \in \mathbb{R}} \int|z-y| F(d z \mid X=x) .
$$

It is easy to get an estimator $\hat{m}(x)$ of $m(x)$ by replacing in (23) $F(d z \mid X=x)$ by an appropriate estimator $\hat{F}(. \mid X=x)$. Then

$$
\hat{m}(x)=\arg \min _{y \in \mathbb{R}} \int|z-y| \hat{F}(d z \mid X=x) .
$$


More details on theoretical and applied properties of median (and more generally on quantiles) can be found in Berlinet et al. (2001) or Gannoun et al. (2003).

Remark 5 The conditional distribution function can be seen as a conditional expectation i.e. $E\left(1_{\{Z \leq z\}} \mid X=x\right)=F(z \mid X=x)$. Morover, if $F(. \mid X=x)$ is continuous and strictly increasing, the conditional median of $Z$ given $X=x$ is obtained as follows :

$$
m(x)=F(0.5 \mid X=x) .
$$

\subsubsection{Median kernel return estimation}

Given $T$ return observations $r_{t}, t=1, \ldots, T$, from a given asset or portfolio, the kernel estimator $\hat{F}\left(z \mid r_{t}\right)$ of the conditional distribution function $F\left(z \mid r_{t}\right)$ is defined by

$$
\hat{F}\left(z \mid r_{t}\right)=\frac{\sum_{l=1}^{T} 1_{\left\{r_{l} \leq z\right\}} K\left(\frac{r_{t}-r_{l}}{h}\right)}{\sum_{l=1}^{T} K\left(\frac{r_{t}-r_{l}}{h}\right)} .
$$

Using Koenker (2005) and (26), a kernel median return estimation is obtained directly by :

$$
\hat{\hat{r}}_{t}=\arg \min _{z \in \mathbb{R}} \frac{\sum_{l=1}^{T}\left|r_{l}-z\right| K\left(\frac{r_{t}-r_{l}}{h}\right)}{\sum_{l=1}^{T} K\left(\frac{r_{t}-r_{l}}{h}\right)} .
$$

Another way to get $\hat{\hat{r}}_{t}$ is to solve the following equation

$$
\hat{F}\left(z \mid r_{t}\right)=\frac{\sum_{l=1}^{T} 1_{\left\{r_{l} \leq z\right\}} K\left(\frac{r_{t}-r_{l}}{h}\right)}{\sum_{l=1}^{T} K\left(\frac{r_{t}-r_{l}}{h}\right)}=\frac{1}{2} .
$$

Remark 6 In the mathematical statistics literature, more complicated nonparametric estimators of conditional median exist : local polynomial method, double kernel method, L-estimator method ...(see, for instance, Gannoun et al. (2002) for more details). It is well-known that the median smooths better than the mean and is less sensitive to the presence of outliers.

\subsubsection{Kernel median and DRS estimation}

Using the robustness of the median, a new estimation of $D S R$ will be proposed. To this end, all the returns observations $r_{j t}, t=1, \ldots, T$ and $j=1, \ldots, m$, are replaced by their median kernel estimators (median regression) $\hat{\hat{r}}_{j t}$, which are a weighted median of the observations.

Let $\omega=\left(\omega_{1}, \omega_{2}, \ldots, \omega_{m}\right)$. The DSR of given portfolio with $m$ assets, is given by

$$
\operatorname{DSR}(\omega)=\frac{1}{T} \sum_{t=1}^{T}\left[\min \left(\hat{\hat{r}}_{p t}-B, 0\right)\right]^{2}, \hat{\hat{r}}_{p t}=\omega_{1} \hat{\hat{r}}_{1 t}+\omega_{2} \hat{\hat{r}}_{2 t}+\ldots+\omega_{m} \hat{\hat{r}}_{m t},
$$

where $\hat{\hat{r}}_{j t}$ is the kernel median estimator of the return $r_{j t}$ of asset $j$ on date $t$. 


\subsubsection{Kernel median and DRS estimation}

Using the robustness of the median, a new estimation of $D S R$ will be proposed. To this end, all the returns observations $r_{j t}, t=1, \ldots, T$ and $j=1, \ldots, n$, are replaced by their median kernel estimators (median regression) $\hat{\hat{r}}_{j t}$, which are a weighted median of the observations.

Let $\omega=\left(\omega_{1}, \omega_{2}, \ldots, \omega_{n}\right)$. The DSR of given portfolio with $n$ assets, is given by

$$
\operatorname{DSR}(\omega)=\frac{1}{T} \sum_{t=1}^{T}\left[\min \left(\hat{\hat{r}}_{p t}-B, 0\right)\right]^{2}, \hat{\hat{r}}_{p t}=\omega_{1} \hat{\hat{r}}_{1 t}+\omega_{2} \hat{\hat{r}}_{2 t}+\ldots+\omega_{n} \hat{\hat{r}}_{n t},
$$

where $\hat{\hat{r}}_{j t}$ is the kernel median estimator of the return $r_{j t}$ of asset $j$ on date $t$.

\section{Nonparametric DSR minimization algorithm}

In this section, the algorithm that minimizes the nonparametric DSR is presented. The idea of this algorithm is to minimize the DSR in a sub-set of dates such that the DSR has a close form solution. The sub-set is then iteratively modified up to global DSR minimization. Finally, a method to construct the efficient frontier is proposed.

\subsection{Case of a portfolio with two assets}

Suppose that they are two risky assets $a$ and $b$ to constitute the portfolio $P$. Let $\omega_{0}$ be a starting portfolio, $\omega_{0}=\left(\omega_{0, a}, \omega_{0, b}\right)=\left(\omega_{0, a}, 1-\omega_{0, a}\right)$ where $\omega_{0, a}$ and $\omega_{0, b}$ are the proportions of the portfolio allocated to assets $a$ and $b$. The return of this portfolio on date $t$ is $r_{0, p t}=$ $\omega_{0} r_{a t}+\left(1-\omega_{0}\right) r_{b t}$. All this returns are replaced with a the kernel median estimators defined above :

$$
\hat{\hat{r}}_{0, p t}=\arg \min _{z \in \mathbb{R}} \frac{\sum_{l=1}^{T}\left|r_{0, p l}-z\right| K\left(\frac{r_{0, p t}-r_{0, p l}}{h}\right)}{\sum_{l=1}^{T} K\left(\frac{r_{0, p t}-r_{0, p l}}{h}\right)} .
$$

Then, for each single asset, the $T$ returns are estimated following the same procedure and using the weighting given to portfolio associated to $\omega_{0}$ :

$$
\hat{\hat{r}}_{0, a t}=\arg \min _{z \in \mathbb{R}} \frac{\sum_{l=1}^{T}\left|r_{a l}-z\right| K\left(\frac{r_{0, p t}-r_{0, p l}}{h}\right)}{\sum_{l=1}^{T} K\left(\frac{r_{0, p t}-r_{0, p l}}{h}\right)}, \hat{\hat{r}}_{0, b t}=\arg \min _{z \in \mathbb{R}} \frac{\sum_{l=1}^{T}\left|r_{b l}-z\right| K\left(\frac{r_{0, p t}-r_{0, p l}}{h}\right)}{\sum_{l=1}^{T} K\left(\frac{r_{0, p t}-r_{0, p l}}{h}\right)},
$$

Consider $S_{0}$ the set of indexes such that the estimated excess portfolio returns associated to $\omega^{(0)}$ are negative

$$
S_{0}=\left\{t \mid 1<t<T \text { such that } \hat{\hat{r}}_{0, p t}<B\right\},
$$

and solve the following problem :

$$
\arg \min _{\omega} \sum_{t \in S_{0}}\left(\hat{\hat{r}}_{p t}-B\right)^{2}, \hat{\hat{r}}_{p t}=\omega \hat{\hat{r}}_{0, a t}+(1-\omega) \hat{\hat{r}}_{0, b t} .
$$

The solution is denoted $\omega_{1}$ and the associated portfolio returns are $r_{1, p t}=\omega_{1} r_{a t}+\left(1-\omega_{1}\right) r_{b t}$. These returns are nonparametrically estimated by :

$$
\hat{\hat{r}}_{1, p t}=\arg \min _{z \in \mathbb{R}} \frac{\sum_{l=1}^{T}\left|r_{1, p l}-z\right| K\left(\frac{r_{1, p t}-r_{1, p l}}{h}\right)}{\sum_{l=1}^{T} K\left(\frac{r_{1, p t}-r_{1, p l}}{h}\right)} .
$$


The returns of each single asset are deduced as follows :

$$
\hat{\hat{r}}_{1, a t}=\arg \min _{z \in \mathbb{R}} \frac{\sum_{l=1}^{T}\left|r_{a l}-z\right| K\left(\frac{r_{1, p t}-r_{1, p l}}{h}\right)}{\sum_{l=1}^{T} K\left(\frac{r_{1, p t}-r_{1, p l}}{h}\right)}, \hat{\hat{r}}_{1, b t}=\arg \min _{z \in \mathbb{R}} \frac{\sum_{l=1}^{T}\left|r_{b l}-z\right| K\left(\frac{r_{1, p t}-r_{1, p l}}{h}\right)}{\sum_{l=1}^{T} K\left(\frac{r_{1, p t}-r_{1, p l}}{h}\right)},
$$

Consider $S_{1}$ the set of indexes such that the estimated excess portfolio returns associated to $\omega^{(1)}$ are negative :

$$
S_{1}=\left\{t \mid 1 \leq t \leq T \text { such that } \hat{\hat{r}}_{1, p t}<B\right\}
$$

and resolve the following optimization problem :

$$
\arg \min _{\omega} \sum_{t \in S_{1}}\left(\hat{\hat{r}}_{p t}-B\right)^{2}, \hat{\hat{r}}_{p t}=\omega \hat{\hat{r}}_{1, a t}+(1-\omega) \hat{\hat{r}}_{1, b t}
$$

where $\hat{\hat{r}}_{1, a t}$ and $\hat{\hat{r}}_{1, b t}$ are defined in $(31)$.

From here on, the same procedure is followed to get $S_{2}, S_{3} \ldots$ Even with the same set of selected observations, iterations should be continued because using the kernel estimations will be different for every new portfolio found. Nevertheless, it should be noted that the changes in the portfolio ( and consequently on DSR), will be smaller from one iteration to another. Thus, iterations should be stopped when changes become smaller than a fixed limit.

4.2 Case of a portfolio with $m$ assets

\subsubsection{The portfolio optimization method}

Suppose that they are $m$ assets available. We start with an arbitrary portfolio $\omega_{0}=\left(\omega_{0,1}, \omega_{0,2}, \ldots, \omega_{0, m}\right)$. For each date $t, t=1, \ldots, T$, the return of this portfolio $r_{0, p t}=\omega_{0,1} r_{1 t}+\cdots+\omega_{0, m} r_{m t}$. All these returns are replaced by median kernel estimators :

$$
\hat{\hat{r}}_{0, p t}=\arg \min _{z \in \mathbb{R}} \frac{\sum_{l=1}^{T}\left|r_{0, p l}-z\right| K\left(\frac{r_{0, p t}-r_{0, p l}}{h}\right)}{\sum_{l=1}^{T} K\left(\frac{r_{0, p t}-r_{0, p l}}{h}\right)}, t=1, \ldots, T .
$$

The estimation of returns of each single asset $i$ is given by

$$
\hat{\hat{r}}_{0, i t}=\arg \min _{z \in \mathbb{R}} \frac{\sum_{l=1}^{T}\left|r_{i l}-z\right| K\left(\frac{r_{0, p t}-r_{0, p l}}{h}\right)}{\sum_{l=1}^{T} K\left(\frac{r_{0, p t}-r_{0, p l}}{h}\right)} ; t=1, \ldots, T, \quad i=1, \ldots, m .
$$

From the previous estimators, dates when the estimated returns of portfolio associated to $\omega_{0}$ had negative excess returns are selected. This set is called $S_{0}$.

Let $\hat{R}_{0, i t}=\hat{\hat{r}}_{0, i t}-B$ and $M_{0}$ the following positive semidefinite matrix :

$$
\begin{gathered}
M_{0}=\frac{1}{T} \sum_{t \in S_{0}}\left[\begin{array}{c}
\hat{R}_{0,1 t} \\
\hat{R}_{0,2 t} \\
\vdots \\
\hat{R}_{0, m t}
\end{array}\right]\left[\hat{R}_{0,1 t} \hat{R}_{0,2 t} \cdots \hat{R}_{0, m t}\right] \\
=\frac{1}{T} \sum_{t \in S_{0}}\left[\begin{array}{ccc}
\left(\hat{R}_{0,1 t}\right)^{2} & \hat{R}_{0,1 t} \hat{R}_{0,2 t} & \hat{R}_{0,1 t} \hat{R}_{0, m t} \\
\hat{R}_{0,2 t} \hat{R}_{0,1 t} & \left(\hat{R}_{0,2 t}\right)^{2} & \hat{R}_{0,2 t} \hat{R}_{0, m t} \\
\vdots & \vdots & \vdots \\
\hat{R}_{0, m t} \hat{R}_{0,1 t} & \hat{R}_{0, m t} \hat{R}_{0,2 t} & \left(\hat{R}_{0, m t}\right)^{2}
\end{array}\right] .
\end{gathered}
$$


The next step is to find the portfolio weight $\omega_{1}$ that solves the following problem :

$$
\arg \min _{\omega} \omega^{\top} M_{0} \omega \text { with } \omega^{\top} \mathbf{1}=1 \text {. }
$$

The solution is given by

$$
\omega_{1}=\left(\omega_{1,1}, \omega_{1,2}, \ldots, \omega_{1, m}\right)=\frac{M_{0}^{-1} \mathbf{1}}{\mathbf{1}^{\top} M_{0}^{(-1)} \mathbf{1}} .
$$

The new portfolio return $r_{1, p t}$ associated to $\omega_{1}$ are

$$
r_{1, p t}=\omega_{1,1} r_{1 t}+\omega_{1,2} r_{2 t}+\ldots \omega_{1, m} R_{m t} .
$$

The associated smooth returns are :

$$
\hat{\hat{r}}_{1, p t}=\arg \min _{z \in \mathbb{R}} \frac{\sum_{l=1}^{T}\left|r_{1, p l}-z\right| K\left(\frac{r_{1, p t}-r_{1, p l}}{h}\right)}{\sum_{l=1}^{T} K\left(\frac{r_{1, p t}-r_{1, p l}}{h}\right)}, \quad t=1, \ldots T,
$$

and the smoothing returns of the assets belonging the portfolio are :

$$
\hat{\hat{r}}_{1, i t}=\arg \min _{z \in \mathbb{R}} \frac{\sum_{l=1}^{T}\left|r_{i l}-z\right| K\left(\frac{r_{1, p t}-r_{1, p l}}{h}\right)}{\sum_{l=1}^{T} K\left(\frac{r_{1, p t}-r_{1, p l}}{h}\right)}, \quad t=1, \ldots, T, \quad i=1, \ldots, m .
$$

Now, let $S_{1}$ be the set of all indices such that $\hat{\hat{r}}_{1, p t}-B<0$, i.e.

$$
S_{1}=\left\{t \mid 1 \leq t \leq T \text { where } \hat{\hat{r}}_{1, p t}-B<0\right\} .
$$

Let $\hat{R}_{1, i t}=\hat{\hat{r}}_{1, i t}-B, t=1, \ldots, T$ and $i=1, \ldots, m$.

Using the above estimators, the following new positive definite matrix $M_{1}$ is built :

$$
\begin{gathered}
M_{1}=\frac{1}{T} \sum_{t \in S_{1}}\left[\begin{array}{c}
\hat{R}_{1,1 t} \\
\hat{R}_{1,2 t} \\
\vdots \\
\hat{R}_{1, n t}
\end{array}\right]\left[\hat{R}_{1,1 t} \hat{R}_{1,2 t} \cdots \hat{R}_{1, n t}\right] \\
=\frac{1}{T} \sum_{t \in S_{1}}\left[\begin{array}{ccc}
\left(\hat{R}_{1,1 t}\right)^{2} & \hat{R}_{1,1 t} \hat{R}_{1,2 t} & \hat{R}_{1,1 t} \hat{R}_{1, m t} \\
\hat{R}_{1,2 t} \hat{R}_{1,1 t} & \left(\hat{R}_{1,2 t}\right)^{2} & \hat{R}_{1,2 t} \hat{R}_{1, m t} \\
\vdots & \vdots & \vdots \\
\hat{R}_{1, m t} \hat{R}_{1,1 t} & \hat{R}_{1, m t} \hat{R}_{1,2 t} & \left(\hat{R}_{1, m t}\right)^{2}
\end{array}\right] .
\end{gathered}
$$

Next step is to to solve the following optimization problem

$$
\arg \min _{\omega} \omega^{\top} M_{1} \omega \text { with } \omega^{\top} \mathbf{1}=1 .
$$

The solution is given by

$$
\omega_{2}=\left(\omega_{2,1}, \ldots, \omega_{2, m}\right)=\frac{M_{1}^{-1} \mathbf{1}}{\mathbf{1}^{\top} M_{1}^{-1} \mathbf{1}} .
$$

Then, following the same procedure, all the returns $r_{2, p t}$ and their smoothing version $\hat{\hat{r}}_{2, p t}$ associated to $\omega_{2}$ are constructed. Similarly, for each asset $i, i=1, \ldots, m$, all its corresponding smoothing returns $\hat{\hat{r}}_{1, i t}, t=1, \ldots, T$ are derived. The iteration should stop when the changes in the portfolio become insignificant, or simply smaller than a pre-established limit. 
Remark 7 From a computational point of view, the iterations are more complicated than the classical DSR because the median kernel estimators of returns change on each step. Nevertheless, the portfolio frontier will be a smoother curve than in the classical case (see below).

To solve the kernel mean DSR minimization problem described in Subsection (2.3.2.1), all the previous steps should be respected with replacing $\hat{\hat{r}}_{s, p t}$ and $\hat{\hat{r}}_{s, i t}$ by $\hat{r}_{s, p t}$ and $\hat{r}_{s, i t}$.

\subsubsection{Nonparametric methods in efficient frontier construction}

The idea is to mix the procedure exhibited in section 2.2.2 with an adapted optimization program. The major change is in the use of the kernel median estimators of returns (or the kernel mean estimators) instead of the observed returns. The aim is to get smooth efficient frontier compared to frontiers determined by classical models (Mean-Variance and Mean-DSR).

Let $M$ the semidefinite semicovariance matrix with coefficients determined by

$$
\Sigma_{i j B}=\frac{1}{T} \sum_{t=1}^{K}\left(r_{i t}-B\right)\left(r_{j t}-B\right),
$$

where periods 1 to $K$ are periods in witch the portfolio under-performs the benchmark $B$.

The problem remains the same as in (3) with additional constraints concerning the weights : positivity and Short-Selling constraints :

1. Positivity constraint

$$
\begin{gathered}
\min _{\omega} \omega^{\top} M \omega \\
\text { subjcet to } \\
\left\{\begin{array}{l}
\omega^{\top} \mathbf{1}=1 \\
\omega^{\top} \mu=E^{*} \\
\omega_{i} \geq 0, i=1,2, \ldots, n
\end{array}\right.
\end{gathered}
$$

2. Short-Selling constraint

$$
\begin{aligned}
& \min _{\omega} \omega^{\top} M \omega \\
& \text { subject to } \\
& \left\{\begin{array}{l}
\omega^{\top} \mathbf{1}=1 \\
\omega^{\top} \mu=E^{*}
\end{array}\right.
\end{aligned}
$$

Same steps as above are followed. On each step $(j)$, until the convergence, we solve the following problem :

$$
\begin{aligned}
& \arg \min _{\omega} \omega^{\top} M_{j} \omega \\
& \text { subjcet to }
\end{aligned}
$$

$$
\begin{array}{ll}
\text { Positivity constraint } & \text { Short-Selling } \\
\omega^{\top} \mathbf{1}=1 & \omega^{\top} \mathbf{1}=1 \\
\omega^{\top} \tilde{\mu}^{j}=E^{*} & \omega^{\top} \tilde{\mu}^{j}=E^{*} \\
\omega_{i} \geq 0, i=1,2 \ldots, m &
\end{array}
$$

where

$$
\tilde{\mu}^{j}=\left[\frac{\sum_{t} \tilde{r}_{j, 1 t}}{T}, \ldots, \frac{\sum_{t} \tilde{r}_{j, m t}}{T}\right]^{\top}
$$


with $\tilde{r}$ represents the nonparametric estimated return obtained by mean or median kernel methods.

After the achievement of the convergence (in $(F+1)$ th step), the minimum DownSide Risk portfolio with expected excess return given by $E^{*}$ will be given by :

$$
\left(\omega_{F+1}\right)^{\top} M_{F} \omega_{F+1} \text {. }
$$

Remark 8 1. Under the Short-Selling constraint, the optimal portfolio weight is obtained by successive iterations (similar approach as (2.2.2)) which leads, thanks to the Lagrangian method, to the following explicit expression :

$$
\omega_{F+1}=\frac{\hat{\alpha} E^{*}-\hat{\lambda}}{\hat{\alpha} \hat{\theta}-\hat{\lambda}^{2}} M_{F}^{-1} \tilde{\mu}^{F}+\frac{\hat{\theta}-\hat{\lambda} E^{*}}{\hat{\alpha} \hat{\theta}-\hat{\lambda}^{2}} M_{F}^{-1} \mathbf{1},
$$

where $\hat{\alpha}=\mathbf{1}^{\top} M_{F}^{-1} \mathbf{1}, \hat{\lambda}=\mathbf{1}^{\top} M_{F}^{-1} \tilde{\mu}^{F}$ and $\hat{\theta}=\left(\tilde{\mu}^{F}\right)^{\top} M_{F}^{-1} \tilde{\mu}^{F}$.

Then the DownSide Risk is :

$$
\operatorname{DSR}\left(\omega_{F+1}\right)=\frac{\alpha\left(E^{*}\right)^{2}-2 \lambda E^{*}+\theta}{\alpha \theta-\lambda^{2}} .
$$

2. In the case of the positivity constraint, the Quad.Prog in R-package is adjusted and adapted to resolve each step of the minimization program.

\section{Empirical analysis}

Interesting applications dealing with Mean-DSR optimization on real data were performed by Zenios and Kang (1993) and Pla-Santamaria and Bravo (2013).

In this section, the performance of the proposed methods are investigated. Classical and nonparametric methods are compared and tested under different kind of constraints : positivity and Short-Selling constraints. It is supposed that there is no transaction costs and no taxes.

\subsection{Data description}

A dataset, drawn from Reuters, was used for this analysis. The original Data consists in daily 9 stocks returns belonged to different sectors : banks, insurance, industry, energy, technology and telecommunication, from the French stock market :

- Nine assets from French Stock market throughout the period from April 2000 to April 2014, yielding a total of 3579 daily observations,

- The CAC 40 (the French Stock Market Index) values (observed in the same period) will provide a baseline for subsequent comparisons between the different methods.

- The one year Government French Bond for 2013 is considered as the Risk-Free Rate of Return.

- The benchmark B is supposed to be equal to zero.

Historical Price Data $p_{t}$ is adjusted for dividends. The assets returns are calculated from stock prices observed on Thomson Reuters Platform as follows :

$$
R_{t}=\frac{p_{t}-p_{t-1}}{p_{t-1}}
$$

with 
- $p_{t}:$ Stock price at date $t$,

- $p_{t-1}:$ Stock price at date $t-1$

The historical statistics of the asset markets are summarised in Table (1)

\begin{tabular}{lcccccc}
\hline & Min & Mean & sd & Skewness & Kurtosis & Max \\
\hline Accor & -0.1294 & 0.000225 & 0.02230 & 0.09481 & 3.0857 & 0.1241 \\
Airb & -0.2632 & 0.000436 & 0.02580 & -0.13740 & 5.5292 & 0.1340 \\
Alcat & -0.1975 & 0.000531 & 0.03607 & 0.45715 & 8.4942 & 0.4054 \\
BNP & -0.1724 & 0.000327 & 0.02601 & 0.65718 & 8.8503 & 0.2090 \\
Carr & -0.1102 & 0.000157 & 0.01977 & 0.12020 & 2.8544 & 0.0995 \\
Dano & -0.1050 & 0.000285 & 0.01571 & 0.09250 & 4.2764 & 0.1019 \\
Oréal & -0.1112 & 0.000261 & 0.01745 & 0.21990 & 4.5650 & 0.1474 \\
S.Gén & -0.1623 & 0.000251 & 0.02890 & 0.39010 & 6.7635 & 0.2389 \\
Total & -0.0919 & 0.0001431 & 0.01716 & 0.22629 & 5.6594 & 0.1364 \\
\hline
\end{tabular}

TABLE 1: Market Historical Statistics

In term of the third moment of expected returns (skewness), Alcatel, BNP, Carrefour, L'Oréal and Société Générale (in the French Market) are positive skews. Accor and Danone returns are approximatively normally distributed. All the distributions have heavier tails and a higher peak than the normal. Therefore, these findings suggest the use of Downside Risk to find the optimal portfolio.

The correlation matrix is given in the Table (2).

\begin{tabular}{lccccccccc}
\hline & Accor & Airb & Alcat & BNP & Carr & Dano & Oréal & S. Gén & Total \\
\hline Accor & 1.000 & 0.457 & 0.447 & 0.544 & 0.474 & 0.351 & 0.426 & 0.539 & 0.489 \\
Airb & 0.457 & 1.000 & 0.401 & 0.418 & 0.404 & 0.337 & 0.376 & 0.407 & 0.415 \\
Alcat & 0.447 & 0.401 & 1.000 & 0.465 & 0.422 & 0.28 & 0.383 & 0.476 & 0.404 \\
BNP & 0.544 & 0.418 & 0.465 & 1.000 & 0.522 & 0.367 & 0.469 & 0.803 & 0.554 \\
Carr & 0.474 & 0.404 & 0.422 & 0.522 & 1.000 & 0.454 & 0.518 & 0.508 & 0.527 \\
Dano & 0.351 & 0.337 & 0.28 & 0.367 & 0.454 & 1.000 & 0.488 & 0.347 & 0.455 \\
Oréal & 0.426 & 0.376 & 0.383 & 0.469 & 0.518 & 0.488 & 1.000 & 0.434 & 0.512 \\
S. Gén & 0.539 & 0.407 & 0.476 & 0.803 & 0.508 & 0.347 & 0.434 & 1.000 & 0.514 \\
Total & 0.489 & 0.415 & 0.404 & 0.554 & 0.527 & 0.455 & 0.512 & 0.514 & 1.000 \\
\hline
\end{tabular}

TABLE 2: French correlation matrix

This matrix shows that the assets are weakly correlated because the stocks belong to different sectors.

\subsection{Analysis of results}

In this section two strategies are investigated : optimization with positive weights and optimization with short selling. In order to ensure clear reading of our results ,from now on, the measures of risk (Variance and DSR) are multiplied by $10^{4}$ in all the tables and graphics exhibiting our simulations.

\subsubsection{Optimization with positive weights}

Here, the optimal portfolio is obtained under positive constraints on the weights which mean that short selling is not allowed $\left(\omega_{i} \geq 0\right.$, for $\left.i=1, \ldots, m\right)$. Four methods are used and tested to 
determine the optimal portfolio.

\section{A. The Mean-Variance method}

The Mean-Variance Markowitz method is used in order to get an optimal portfolio. Assets distribution is supposed to be symmetric. The initial data is divided into two parts : one for making optimization and one for testing the efficiency of the method. The test sample is used to assess performance against the CAC 40 index during one year (2013).

\section{A.1 The efficient frontier}

The efficient frontier obtained with the Markowitz method is displayed in Figure 2.

Figure 2: Markowitz Mean-Variance efficient frontier.

Figure 2 shows the efficient frontier represented by the upper leg of the hyperbola. The minimum risk portfolio is given by the vertex $(0.104,0.03)$. To put it clearly, Table 3 gives the optimal portfolio for many expected returns $E^{*}$.

\begin{tabular}{ccccccccccc}
\hline$E^{*}(\%)$ & Variance & $\omega_{1}$ & $\omega_{2}$ & $\omega_{3}$ & $\omega_{4}$ & $\omega_{5}$ & $\omega_{6}$ & $\omega_{7}$ & $\omega_{8}$ & $\omega_{9}$ \\
\hline 0.04 & 0.1055 & 0 & 0.1024 & 0 & 0 & 0 & 0.4379 & 0.3454 & 0 & 0.1143 \\
\hline 0.05 & 0.1087 & 0 & 0.1554 & 0 & 0 & 0 & 0.4318 & 0.4083 & 0 & 0.0045 \\
\hline 0.06 & 0.1169 & 0 & 0.1554 & 0 & 0 & 0 & 0.3104 & 0.4291 & 0 & 0 \\
\hline 0.07 & 0.1327 & 0 & 0.3677 & 0 & 0 & 0 & 0.1841 & 0.4482 & 0 & 0 \\
\hline
\end{tabular}

TABLE 3: Weights for optimal Portfolios using Mean-Variance method.

One can observe that, the assets Airbus, Danone and L'Oréal are part of the optimal portfolios for all level of expected returns. It is well-known that those companies are financially strong. Total asset contributes to the optimal portfolio only for low expected returns.

\section{A.2 Portfolio returns against $C A C 40$}

Now, for a given expected return $\left(E^{*}=0.05 \%\right)$, the return of the optimal portfolio is compared to the French CAC 40 index. A total of 239 portfolio returns (in working market days during 2013) are compared to 239 CAC 40 daily values. In $52 \%$ of cases, the optimal portfolio performed relatively better than the CAC40 index. The same conclusion is reached for different choices of $E^{*}$. The obtained daily returns during the test period are drawn in Figure 3.

Figure 3: Portfolio returns against CAC 40 Index.

\section{B. The DSR methods}

Following the same principle as before, three methods are applied on the previous data (classical DSR, DSR with mean smoothing, DSR with median smoothing). For nonparametric methods, 
the kernel density used is the Gaussian one :

$$
K(x)=\frac{1}{\sqrt{2 \pi}} \exp \left(\frac{-x^{2}}{2}\right)
$$

The bandwidths are determined by the following cross-validation method.

$$
h_{o p}=\arg \min _{h} C V(h)=\arg \min _{h} \sum_{i=1}^{T}\left[R_{p i}-\hat{R}_{(-i, p i)}(h)\right]^{2}
$$

where

— for mean smoothing

$$
\hat{R}_{(-i, p i)}(h)=\frac{\sum_{t=1, t \neq i}^{T} R_{p t} K\left(\frac{R_{p i}-R_{p t}}{h}\right)}{\sum_{t=1, t \neq i}^{T} K\left(\frac{R_{p i}-R_{p t}}{h}\right)},
$$

— for median smoothing

$$
\hat{R}_{(-i, p i)}(h)=\arg \min _{z \in \mathbb{R}} \frac{\sum_{t=1, t \neq i}^{T}\left|R_{p t}-z\right| K\left(\frac{R_{p i}-R_{p t}}{h}\right)}{\sum_{t=1, t \neq i}^{T} K\left(\frac{R_{p i}-R_{p t}}{h}\right)} .
$$

The general shape of $C V($.$) curve is exhbited in Figure (4)$

Figure 4: Example of CV(h)

\section{B.1 The efficient frontiers}

Figure 5 displays the efficient frontier curves. The first part of this graphic allows easy comparison between the three proposed Methods. The second part is devoted exclusively to the median method.

Figure 5: DSR efficient frontiers.

It is apparent from the graphical comparison in Figure 5. that nonparametric methods push the efficient frontiers outward to the left of the classical DSR curve to produce efficient portfolios that are stochastically dominant.

In terms of DSR, the nonparametric methods have the smallest values for a given expected return $E^{*}$. The method based on Median smoothing is more efficient. Table 4 gives an overview of optimal portfolios obtained by each method for various expected returns $E^{*}$.

From now on, the abbreviations below will be used :

- $\omega_{i}, i=1, \ldots, 9:$ the optimal weights,

- I : Classical DSR,

- II : DSR with mean smoothing,

— III : DSR with median smoothing. 


\begin{tabular}{cccccccccccc}
\hline Methods & $E^{*}(\%)$ & DSR & $\omega_{1}$ & $\omega_{2}$ & $\omega_{3}$ & $\omega_{4}$ & $\omega_{5}$ & $\omega_{6}$ & $\omega_{7}$ & $\omega_{8}$ & $\omega_{9}$ \\
\hline I & 0.04 & 0.049 & 0 & 0.064 & 0 & 0 & 0 & 0.448 & 0.425 & 0 & 0.062 \\
II & 0.04 & 0.010 & 0 & 0.000 & 0 & 0 & 0 & 0.780 & 0.211 & 0 & 0.000 \\
III & 0.04 & 0.002 & 0 & 0.506 & 0 & 0 & 0 & 0.000 & 0.000 & 0 & 0.493 \\
\hline I & 0.05 & 0.050 & 0 & 0.138 & 0 & 0 & 0 & 0.384 & 0.477 & 0 & 0.000 \\
II & 0.05 & 0.012 & 0 & 0.310 & 0 & 0 & 0 & 0.689 & 0.000 & 0 & 0.000 \\
III & 0.05 & 0.004 & 0 & 0.567 & 0 & 0 & 0 & 0.000 & 0.432 & 0 & 0.000 \\
\hline I & 0.06 & 0.052 & 0 & 0.243 & 0 & 0 & 0 & 0.249 & 0.507 & 0 & 0.000 \\
II & 0.06 & 0.014 & 0 & 0.394 & 0 & 0 & 0 & 0.605 & 0.000 & 0 & 0.000 \\
III & 0.06 & 0.006 & 0 & 0.567 & 0 & 0 & 0 & 0.459 & 0.000 & 0 & 0.000 \\
\hline I & 0.07 & 0.059 & 0 & 0.348 & 0 & 0 & 0 & 0.115 & 0.536 & 0 & 0.000 \\
II & 0.07 & 0.024 & 0 & 0.439 & 0 & 0 & 0 & 0.283 & 0.277 & 0 & 0.000 \\
III & 0.07 & 0.012 & 0 & 0.762 & 0 & 0 & 0 & 0.238 & 0.000 & 0 & 0.000 \\
\hline
\end{tabular}

TABLE 4: Weights for optimal portfolios using DSR methods.

There is a specific strategy for each method. Although, for the three methods, the assets Alcatel, BNP and Carrefour do not contribute to the optimal portfolio for different level of expected return, the contribution of the other assets are different from one method to another. In term of DSR, the nonparametric methods are more efficient than the classical DSR method. The nonparametric method based on median estimation has the smallest DSR for all the expected returns.

\section{B.2 Portfolio returns against $C A C 40$}

To compare the efficiency of these methods, the daily French CAC 40 index is used. The daily portfolio returns are computed using the weights obtained with the sample data deprived of 2013's observations. Results are summarized in Figure 6.

Figure 6: Portfolio returns against CAC 40 Index.

The portfolio returns obtained by nonparametric methods are equal or slightly better than the CAC 40 index. More precisely, the method using the smoothing median performs better in $54 \%$ of cases. Classical DSR method is less efficient than the CAC 40 (in $47 \%$ of days).

Other indices, mainly Sharpe and Sortino Ratios, are used here to measure numerically the performance of each method. These tools will be exhibited later in this paper.

\subsubsection{Optimization with short selling constraint}

Short-selling is an interesting strategy because it provides a way to speculate if the market's value is going to decline. This allows to add value to the portfolio even in a bear market. Short-selling could be also used to hedge long positions. Concretely, the strategy involves borrowing a stock from a broker and then selling it in the market. The stock is bought back and returned to the broker at a later date, this is called covering the short. If the stock drops, the short seller buys it back at a lower price and then he makes money.

In this section, the optimal portfolio is obtained under realistic constraints, in the sense that short selling is allowed $\left(\omega_{i} \in \mathbb{R}\right.$, for $\left.i=1, \ldots, 9\right)$. Short positions should be more than $-20 \%$ fulfilling the well-known 120-20 strategy in order to limit the possible high losses. The four methods exhibited above will be used and tested to determine the optimal portfolio. 


\section{A. Mean-Variance method}

The classical Mean-Variance model of Markowitz, under short selling constraints , is used in order to get an optimal portfolio. Assets return distribution is supposed to be Gaussian . As the previous section, The initial data is divided into two parts : the first one for determining optimal portfolios and the second one for testing the efficiency of the method. The last sample will be used to assess performance of the optimal portfolio returns against the CAC 40 index measured during one year (2013).

\section{A.1 The efficient frontier}

The efficient frontier with Short-selling constraint is plotted in Figure 7. It is graphically presented as a branch of hyperbola, and the minimum risk portfolio is given by the vertex $(0.105,0.05)$.

FiguRE 7: Mean-Variance efficient frontier with short-selling constraint.

Table 5 shows the optimal portfolio's weights for a given levels of expected return $E^{*}$. Since short positions are allowed, investors are exposed to higher level of risk. Therefore, high level of expected return is required.

\begin{tabular}{ccccccccccc}
\hline$E^{*}(\%)$ & Var & $\omega_{1}$ & $\omega_{2}$ & $\omega_{3}$ & $\omega_{4}$ & $\omega_{5}$ & $\omega_{6}$ & $\omega_{7}$ & $\omega_{8}$ & $\omega_{9}$ \\
\hline 0.17 & 0.314 & -0.158 & 0.917 & -0.146 & -0.005 & -0.20 & -0.014 & 0.893 & -0.087 & -0.20 \\
\hline 0.18 & 0.354 & -0.175 & 0.996 & -0.167 & -0.005 & -0.20 & -0.014 & 0.893 & -0.087 & -0.20 \\
\hline 0.19 & 0.399 & -0.192 & 1.075 & -0.188 & -0.048 & -0.20 & -0.148 & 0.954 & -0.096 & -0.20 \\
\hline 0.20 & 0.447 & -0.20 & 1.163 & -0.20 & -0.011 & -0.20 & -0.20 & 0.955 & -0.106 & -0.20 \\
\hline
\end{tabular}

TABLE 5: Optimal portfolios using Mean-Variance method.

Investors should buy the assets AIRBUS and L'OREAL and take short positions for all the other assets to construct the optimal portfolios, which means that, in that period French Stock Market was Bearish because of the launch of financial transaction taxes, also because of the economic recessions or depressions, when pessimism prevails.

\section{A.2 Portfolio returns against CAC 40}

For a given expected return $\left(E^{*}=0.17 \%\right)$, the return of the optimal portfolio is compared to the French CAC 40 index. In Figure 8, a total of 239 portfolio returns (in working market days during 2013) are compared to 239 CAC 40 daily values. In $51 \%$ of cases, the optimal portfolio returns performed relatively better than the CAC 40 index. The same conclusion is reached for different choices of $E^{*}$. 


\section{B. The DSR methods}

After imposing Short-Selling constraint, the three methods (classical DSR, DSR with mean smoothing, DSR with median smoothing) are applied on the data in order to get optimal portfolios. The same procedure of the previous section is followed.

\section{B.1 The efficient frontiers}

Figure 9 shows the efficient frontier curves for the three proposed methods. The first part of the graphic compares the three Methods. The second part presents only the median method.

Figure 9: DSR efficient frontiers.

Compared to the classical Mean-DSR model, the nonparametric methods present low downside risk. More precisely, the nonparametric efficient frontier based on median estimation stochastically dominates the other curves for any given expected return. These analyses are confirmed by results shown in Table 6 which also gives the optimal portfolio weights for different expected returns $E^{*}$.

\begin{tabular}{lccccccccccc}
\hline Mds & $E^{*}(\%)$ & Var & $\omega_{1}$ & $\omega_{2}$ & $\omega_{3}$ & $\omega_{4}$ & $\omega_{5}$ & $\omega_{6}$ & $\omega_{7}$ & $\omega_{8}$ & $\omega_{9}$ \\
\hline I & 0.17 & 0.135 & -0.162 & 0.893 & -0.134 & 0.131 & -0.200 & -0.13 & 1.002 & -0.200 & -0.20 \\
II & 0.17 & 0.010 & -0.200 & 1.460 & -0.200 & -0.200 & 0.501 & -0.20 & 0.239 & -0.200 & -0.20 \\
III & 0.17 & 0.008 & -0.020 & 1.353 & -0.200 & -0.200 & -0.200 & -0.20 & 0.823 & 0.024 & -0.20 \\
\hline I & 0.18 & 0.152 & -0.179 & 0.975 & -0.152 & 0.127 & -0.200 & -0.20 & 1.029 & -0.200 & -0.20 \\
II & 0.18 & 0.015 & -0.200 & 1.571 & -0.200 & -0.200 & 0.490 & -0.20 & 0.139 & -0.20 & 0 \\
III & 0.18 & 0.012 & -0.200 & 1.510 & -0.200 & -0.200 & -0.200 & -0.20 & 0.666 & 0.024 & -0.20 \\
\hline I & 0.19 & 0.171 & -0.200 & 1.066 & -0.177 & 0.119 & -0.200 & -0.20 & 0.992 & -0.200 & -0.20 \\
II & 0.19 & 0.021 & -0.200 & 1.683 & -0.200 & -0.200 & 0.478 & -0.20 & 0.039 & -0.200 & -0.20 \\
III & 0.19 & 0.025 & -0.200 & 1.668 & -0.200 & -0.200 & -0.200 & -0.20 & 0.508 & 0.024 & -0.20 \\
\hline I & 0.20 & 0.191 & -0.200 & 1.161 & -0.200 & 0.100 & -0.200 & -0.20 & 0.939 & -0.200 & -0.20 \\
II & 0.20 & 0.028 & -0.200 & 1.794 & -0.200 & -0.200 & -0.466 & -0.20 & -0.061 & -0.200 & -0.20 \\
III & 0.20 & 0.032 & -0.200 & 1.826 & -0.200 & -0.200 & -0.200 & -0.20 & 0.351 & 0.023 & -0.20 \\
\hline
\end{tabular}

TABLE 6: Weights for optimal portfolios using DSR methods

The three methods propose different Long-Short strategies of investment. In order to take profit of the market evolution, investors should take long position on AIRBUS and L'OREAL assets, and should take short positions on the other assets. It should be noted that, for $0.2 \%$ expected return, L'OREAL asset should be sold when the method based on mean estimation is used. The difference in asset allocations, between the models, reflects the difference in the measure of risk used in the optimization programs.

It is still interesting to note that the nonparametric methods seems appealing, because they have lower risk comparing to the classical Mean-DSR model. The nonparametric method based on median estimation, clearly outperforms the other models in the total return dimension for the entire calculation period . 


\section{B.2 Portfolio returns against CAC 40 Index}

One way of comparing the performance of these methods, is to investigate expost daily optimal portfolio returns. This has been done by using the minimum Semivariance determined by the classical and the nonparametric models, presented previously. The optimal portfolio return is then calculated for the entire data for 2013's keeping asset weight within the portfolios static. Figure 10 shows the obtained portfolio returns as well as the CAC 40 index. The optimal portfolio returns are very close de the CAC 40 index. Accurately, the method using the smoothing median outperforms the CAC 40 index in $55 \%$ of cases.

Figure 10: DSR efficient frontiers.

\subsubsection{Measures of Performance}

Another way to compare the performance of the proposed methods : the parametric and nonparametric methods, is to compute performance ratios : the Sharpe (ShR) and Sortino (SoR) ratios.

- Sharpe Ratio (Sharpe (1966))

The Sharpe ratio is quite simple, which lends to its popularity. The idea of the ratio is to see how much additional return you are receiving for the additional volatility of holding the risky asset over a risk-free asset, then, higher level of Sharpe ratio implies better performance of the portfolio. The Sharpe ratio is given by this formula:

$$
S h R=\frac{E\left(R_{p}\right)-R_{f}}{\sigma\left(R_{p}\right)}
$$

where $E\left(R_{p}\right)$ denotes the expected return of the portfolio, $R_{f}$ the return on the risk-free asset and $\sigma\left(R_{p}\right)$ the standard deviation of the portfolio returns.

This ratio is a relevant performance measure when returns are normally distributed. Abnormalities like kurtosis, fatter tails and higher peaks, or skewness on the distribution can be a problematic for the ratio, as standard deviation doesn't have the same effectiveness when these problems exist. That is why we propose to calculate the Sortino ratio.

- Sortino Ratio (Sortino (1994)) :

Sortino ratio is a transformation of the Sharpe ratio that differentiates harmful volatility from general volatility by taking into account the downside deviation. The use of this ratio is recommended when we analyse highly volatile portfolios. A portfolio is considered as efficient one when it has high Sortino ratio. The Sortino ratio is given by this formula :

$$
S o R=\frac{E\left(R_{p}\right)-\tau}{D S R}
$$

where $E\left(R_{p}\right)$ is the asset or portfolio average realized return, $\tau$ the target or required rate of return for the investment strategy under consideration (originally called the minimum acceptable return) and $D S R$ the target semi-deviation (the square root of target Semivariance), termed downside deviation. 
The obtained Sharpe and Sortino ratios for all methods are given in Table 7 sums up the level of Sharp and Sortino ratios for all the methods.

\begin{tabular}{|c|c|c|c|c|c|c|c|c|}
\hline \multirow{2}{*}{$E^{*}(\%)$} & \multicolumn{4}{|c|}{ Sharpe Ratio } & \multicolumn{4}{|c|}{ Sortino Ratio } \\
\hline & $\mathrm{M}-\mathrm{V}$ & $\overline{\mathrm{DSR}}$ & Mean & Median & $\mathrm{M}-\mathrm{V}$ & DSR & Mean & Median \\
\hline & \multicolumn{8}{|c|}{ Positive weights only } \\
\hline 0.0 & 021 & 0.051 & 0.058 & 0.132 & 0.022 & 0.053 & 0.060 & 0.137 \\
\hline 0.0 & 067 & 0.068 & 0.077 & 0. & 0.069 & 0.069 & 0.079 & 0.115 \\
\hline 0.0 & 0.091 & 0.092 & 0.094 & 0.1 & 0.096 & 0.096 & 0.097 & 0.126 \\
\hline $0.0^{\prime}$ & 110 & 0.111 & 0.118 & 0.142 & 0.116 & 0.117 & 0.122 & 0.157 \\
\hline & \multicolumn{8}{|c|}{ Allowing negative weights } \\
\hline 0.0 & 0.025 & 0.055 & 0.063 & 0.125 & 0.003 & 0.058 & 0.065 & 0.122 \\
\hline & 0.073 & 0.072 & 0.081 & & 0.073 & 0.074 & 0.083 & 0.150 \\
\hline 0 & 0.095 & 0.096 & 0.098 & 0 & 0.100 & 0.101 & 0.102 & 0.130 \\
\hline 0.07 & 0.114 & 0.116 & 0.122 & 0.145 & 0.120 & 0.121 & 0.127 & 0.160 \\
\hline
\end{tabular}

TABle 7: Performance ratios.

Based on those indices, one can observe that nonparametric methods, especially the one using DSR with median smoothing is more efficient than the classical ones. It is interesting to note that the four methods with Short-Selling constraint, except when the expected return $E^{*}=0.04$, have higher Sharpe and Sortino ratios, which means that long-short strategies are more efficient then the long strategy only.

\section{Conclusion}

The classical Mean-Variance model of Markowitz is one of the most commonly used models for determining optimal portfolio weight. However, built on the strict assumption that asset returns are normally distributed, the Mean-Variance is inadequate to optimize portfolio when returns have asymmetric distribution. The traditional Mean-Variance model which treats both the above and the below target returns equally, tends to over estimate the risks .

In reality, investors are more concerned and averse about the downside movements, that is why it is better to find a more appropriate measure of risk. Our contribution consists on the introduction of nonparametric approach in the estimation of the DownSide Risk. It leads to get smoother efficient frontiers. In addition, adequate iterative algorithms were developed to resolve the optimization problems. Nonparametric methods, specially the Mean-DSR with median smoothing, provide lower portfolio risk compared to the classical Mean-Variance and Mean-Semivariance models.

The proposed DownSide Risk versions were applied to determine the optimal weights for 9 assets from the French Stock Market. The results show that the strategies of investment, in terms of asset allocation depend on the methods, and the risk measure used to optimize the portfolio. Asset allocation depends also on the kind of constraints used in the optimisation program like allowing Short-Selling or not.

In fact, The nonparametric methods present lower risk comparing to the classical Mean-Variance and Classical Mean-DSR models. In term of performance, the nonparametric methods, specially the one based on median estimation outperforms the CAC 40 in $52 \%$ for long only strategy, and in 54\% for long-short strategy. They also have higher level of Sharpe and Sortino ratios than the classical methods. It is interesting to note that, long-short strategies are more efficient when we have high expected returns. 
Our empirical studies were limited to the French Stock Market, it will be interesting to test our methods on other assets (Bonds, Options,...) and other markets ( developed and emergent markets). The nonparametric methods give an efficient and simple alternative approach to implement in order to determine the optimal portfolios. The method based on median estimation are precise and very important in terms of performance thanks to the robustness of the median. Nevertheless, computational time is very long. It could be reduced by another optimization algorithm which will be the subject of the ongoing paper.

\section{References}

1. Ang, J., A note on the ESL Portfolio Selection Model, Journal of Financial and Quantitative Analysis, 10, 849-857 (1975).

2. Athayde, G., Building a Mean-Downside Risk Portfolio Frontier, Developments in Forecast Combination and Portfolio Choice, John Wiley and Sons (2001)

3. Athayde, G., The mean-downside risk portfolio frontier : a non-parametric approach, Published in Advances in portfolio construction and implementation (2003).

4. Berlinet, A., Cadre, B., Gannoun, A., On the conditional $L_{1}$-median and its estimation, Journal of Nonparametric Statistics, 13, 631-645 (2001).

5. Berlinet, A., Gannoun, A., Matzner-Lober, E., Asymptotic normality of convergent estimates of conditional quantiles, Statistics, 35, 139-169 (2001)

6. Estrada, J., Mean-Semivariance Behavior : An Alternative Behavioral Model, Journal of Emerging Market Finance, 3, 231-248 (2004).

7. Estrada, J., Semivariance Optimization : A Heuristic Approach, Journal of Applied Finance, 57-72 (2008).

8. Gannoun, A, Saracco, J., Yu, K., Nonparametric time series prediction by conditional median and quantiles, Journal of statistical Planning and inference, 117, 207-223 (2003).

9. Harlow, V., Asset allocation in a downside risk framework, Financial Analyst Journal, 47, 28-40 (1991).

10. Hogan, W. and Warren, J., Computation of the efficient Boundary in the ES Portfolio selection, Journal of Financial and Quantitative Analysis, 9, 1-11 (1974).

11. King, A-J., Asymmetric risk measures and tracking models for portfolio optimization under uncertainty. Annals of Operations Research, 45, 1, 165-177 (1993).

12. Levy, H., Markowitz, H.M., Approximating expected utility by a function of mean and variance, American Economic Review, (3), 308-317 (1979).

13. Markowitz, M., Peter,T., Ganlin, X. and Yuji,Y., Computation of mean-semivariance Efficient sets by the Critical Line Algorithm, Annals of Operations Research, 45, 307-317 (1993).

14. Mamoghli, C. and Daboussi, S., Optimisation de portefeuille downside risk, Social Science Research Network, 23 pages (2008).

15. Markowitz, H., Portfolio Selection, Journal of Finance, 7, 77-91 (1952).

16. Markowitz, H.M., Portfolio Selection : Efficient Diversification of Investments, New York : John Wiley \& Sons (1959).

17. Markowitz, H.M., Portfolio Selection : Efficient Diversification of Investments, Basil Blackwell (1991).

18. Pagan, A.R. and Ullah, A., Nonparametric Econometrics, Cambridge University Press (1999).

19. Pla-Santamaria, D., , Bravo, M., Portfolio optimization based on downside risk: a mean-semivariance efficient frontier from Dow Jones blue chips. Annals of Operations Research, 205, 1, 189-201 (2013).

20. Sharpe, W., Mutual Fund Performance, Journal of Business, 39, 119-138 (1966).

21. Silverman, B.W., Density Estimation for Statistics and Data Analysis, Chapman and Hall, New York (1986).

22. Sortino, F. and L. Price, Performance measurement in a DownSide risk framework, Journal of Investing, 3 , 59-65 (1994).

23. Zenios, S-A., Kang, P., Mean-absolute deviation portfolio optimization for mortgage-backed securities. Annals of Operations Research, 45, 1, 433-450 (1993). 


\section{Annexe 1}

Let $\omega=\left(\omega_{1}, \omega_{2}, \ldots, \omega_{m}\right)^{\top}, \mu_{j}=\overline{r_{j}}, j=1, \ldots, n$ and $\mu=\left(\mu_{1}, \ldots, \mu_{m}\right)^{\top}$. The Lagrangian method to solve

$$
\begin{gathered}
\operatorname{Min}_{\omega} \omega^{\prime} M \omega \\
\operatorname{under}\left\{\begin{array}{l}
\sum_{j=1}^{m} \omega_{j}=1 \\
\sum_{j=1}^{m} \omega_{j} \mu_{j}=E^{*}
\end{array}\right.
\end{gathered}
$$

is the following: Lagranian $L$ is

$$
L=\frac{1}{2} \omega^{\top} M \omega-\lambda\left(\mu^{\top} \omega-E^{*}\right)-\gamma\left(\mathbf{1}^{\top} \omega-1\right) .
$$

Second order conditions are satisfied because $M$ is definite positive. The first order conditions are

$$
\frac{\partial L}{\partial \omega}=M \mu-\lambda \mu-\gamma \mathbf{1}=0
$$

then

$$
\gamma=\lambda M^{-1} \mu+\gamma M^{-1} \mathbf{1}
$$

By replacing in the two constraints, one get:

$$
\left\{\begin{array}{l}
\lambda A+\gamma B=E^{*} \\
\lambda B+\gamma C=1
\end{array}\right.
$$

where

$$
A=\mu^{\top} M^{-1} \mu, B=\mu^{\top} M^{-1} \mathbf{1}, C=\mathbf{1}^{\top} M^{-1} \mathbf{1}, D=A C-B^{2} .
$$

The optimal vector weights is then given by

$$
\omega^{*}=\frac{C E^{*}-B}{D} M^{-1} \mu+\frac{A-B E^{*}}{D} M^{-1} \mathbf{1} .
$$

